\title{
IDENTIFICACIÓN DE INDICADORES DE CONSERVACIÓN PARA LA RESERVA NACIONAL DE JUNÍN, PERU
}

\section{IDENTIFICATION OF CONSERVATION INDICATORS FOR THE NATIONAL RESERVE OF JUNIN, PERU}

\author{
Claudia Caro ${ }^{1}$, Zulema Quinteros ${ }^{1}$ y Verónika Mendoza²
}

\section{Resumen}

La Reserva Nacional de Junín (RN Junín), considerada como Humedal de Importancia Internacional, especialmente como Hábitat de Aves Acuáticas (Sitio RAMSAR), viene atravesando por una serie de problemas que están causando malestar entre los habitantes de la zona. Teniendo en cuenta este aspecto y en respuesta a las necesidades de información que ayuden a los tomadores de decisiones a cumplir con los objetivos de creación de la Reserva, se identificó un juego de 24 indicadores para monitorear los procesos de conservación que se lleven a cabo en este lugar. Los indicadores fueron elegidos tomando en cuenta las metas y objetivos socialmente determinados y las características que presenta el ecosistema, con la finalidad de evaluar el estado, presión, impacto y respuesta de las actividades humanas sobre los recursos y servicios más importantes de esta Área Natural Protegida. A través de este trabajo se pretende dar a conocer una metodología que permita un efectivo acercamiento de la sociedad a los procesos de monitoreo, en el marco del manejo adaptativo de ecosistemas, resaltando los valores sociales sin dejar de lado el rigor científico de los parámetros ambientales.

Palabras claves: Indicadores, procesos participativos, manejo adaptativo, conservación, Reserva Nacional de Junín

\begin{abstract}
The National Reserve of Junin, considered a RAMSAR site is suffering different kinds of problems that are causing worry among local inhabitants. Taking this into consideration and responding to needs of information to help stakeholders in managing this important natural area, a kit of 24 indicators were determined in order to be used in the follow up conservation processes developed in the area. Indicators were built taking into consideration aims and objectives determined by local inhabitants as well as the main characteristics of the Reserve. This was done in order to value state, stress, impact and response of human activities on the most important natural resources and environmental services of this protected natural area. This research pretended to generate a methodology that can link society and environmental parameters using the adaptative ecosystem management framework, which highlights both social values and scientific aspects of the environment.
\end{abstract}

Key words: Indicators, participative processes, adaptative management, conservation, Nacional Reserve of Junin

\section{Introducción y antecedentes}

El territorio altoandino, ubicado por encima de los 3800 msnm (Grupo en páramos, punas y jallqas del Perú, 2002) brinda bienes y servicios ambientales importantes para sus habitantes y para las zonas bajas (Miller, 1994), tal como sucede en la Reserva Nacional de Junín. En este sentido, se ha desarrollado el presente trabajo de investigación con el objetivo de mejorar el conocimiento acerca de lo que sucede en la zona altoandina, y en especial en la RN Junín, poniendo énfasis en la identificación participativa de indicadores que permitan hacer un adecuado seguimiento a las acciones de conservación que se desarrollen en este lugar.

Los indicadores identificados permitirán evaluar el estado, presión e impacto de las actividades humanas sobre la Reserva, al igual que la capacidad de respuesta de los habitantes frente a los problemas que se presentan, teniendo en cuenta en todo momento la conservación de la integridad de los ecosistemas que componen esta zona (De Leo \& Levin, 1997) y partiendo de la propuesta de reporte de integridad de ecosistemas planteada por Harwell (1999) en la que se consideran los siguientes aspectos: Fijar metas con la sociedad, desarrollar objetivos para cada meta planteada, trasladar los objetivos en características ecológicas esenciales y sus subcategorías, determinar variables referenciales para cada subcategoría y asociarlas con medidas específicas.

En la selección y priorización de los indicadores, se han tomado en cuenta también a las especies clave para el ecosistema (Davic, 2003) y las especies 
culturalmente claves para las personas (Garibaldi \& Turner, 2004), así como la teoría del ciclo adaptativo en la que se postula que un pequeño juego de plantas, animales y procesos abióticos estructuran los ecosistemas a través de escalas de tiempo y espacio diferentes, bajo una concepción cíclica que involucra el movimiento de un sistema a través de cuatro fases: Reorganización, conservación, explotación y liberación (Gunderson \& Holling, citados por Walker et al., 2002).

La identificación de indicadores para monitorear la conservación de la Reserva busca ser un insumo para el manejo adaptativo de este ecosistema, dando prioridad a sus componentes esenciales y asegurando la participación de la sociedad (Schiller et al., 2001) que será la encargada de utilizar la información generada $y$ la que posee los conocimientos específicos acerca del ambiente que quieren desarrollar (Pintér et al., 2000). En un activo manejo adaptativo donde las acciones son diseñadas como experimentos, el componente social mejora el aprendizaje y la novedad para incrementar la resiliencia (Walker et al., 2002). Se debe recordar que ninguna propuesta podrá llevarse a cabo legítimamente y con el compromiso de las personas involucradas, si es que éstas no han participado en el proceso desde el inicio.

A través de esta investigación se espera brindar un alcance para la construcción de indicadores que permitan monitorear la conservación de la integridad de los ecosistemas, usando una metodología sencilla que involucra a los actores sociales en el proceso.

\section{Materiales y Métodos}

Área de Estudio

El Área de estudio es la Reserva Nacional de Junín, ubicada sobre la Meseta de Bombón en los distritos de Carhuamayo, Ondores y Junín en el departamento de Junín y los distritos de Ninacaca y Vicco en el departamento de Pasco ( $10^{\circ} 50^{\prime}$ y $50^{\prime \prime}$ y $11^{\circ} 09^{\prime} 55^{\prime}$ ' LS y $75^{\circ} 59^{\prime}$ y $25^{\prime}$ y $76^{\circ} 15^{\prime} 40^{\prime}$ ' LW) con una extensión de 53000 ha y comprendida entre 4 080 y 4125 msnm (INRENA, 2000).

La temperatura en esta zona oscila entre los $3^{\circ} \mathrm{y}$ $7^{\circ} \mathrm{C}$, siendo los meses más fríos entre Mayo y Septiembre. Anualmente llueve un promedio de 940 mm, siendo los meses de Diciembre a Abril los más lluviosos. La vegetación de la zona se presenta bajo asociaciones naturales como pajonales densos, bofedales u oconales, césped de puna y totorales. La fauna está representada por peces, anfibios, aves y mamíferos, destacando la "rana de Junín" Batrachophrynus macrostomus y "zambullidor de Junín” Podiceps taczanoswkii por ser especies endémicas y en peligro de extinción.

En el aspecto social, la Reserva Nacional de Junín alberga aproximadamente a 46000 personas distribuidas en cinco distritos: Carhuamayo, Junín, Ondores, Vicco y Ninacaca, quienes se dedican básicamente a la ganadería de ovinos, destacando además otras actividades económicas como la minería y el turismo (Vásquez \& Vegas, 1985).

Sin embargo, pese a su gran cantidad de riquezas, la Reserva Nacional de Junín atraviesa diversos problemas que datan de hace muchas décadas, siendo la contaminación minera, la regulación de las aguas mediante la presa de Upamayo y el poco oxígeno disponible en el Lago Junín, los más significativos. A estos problemas se suman también la quema de totorales, el arrojo de efluentes domésticos de las poblaciones aledañas, el pastoreo intensivo, la caza de especies de fauna silvestre como aves y batracios, la extracción de champa y el efecto visual negativo, a causa de la contaminación, que afecta la actividad turística en la Reserva (INRENA, 2000).

Metodología

Recopilación y análisis de información relacionada a la $\mathrm{RN}$ Junín a través de revisión bibliográfica, entrevistas a autoridades políticas y ambientales de la zona, aplicación de encuestas y desarrollo de un taller participativo con autoridades locales y representantes de las comunidades que ocupan la Reserva.

Identificación de actores sociales clave y priorización de problemas de la zona, desde la perspectiva de estos actores, considerando como criterio de selección la frecuencia con la que fue mencionado cada problema.

Teniendo en cuenta los problemas priorizados se definieron metas y objetivos para cada uno de ellos, y se identificaron los servicios ambientales esenciales que brinda la Reserva, considerando para ello la opinión de las personas. Asimismo se determinaron los conductores de cambio del ecosistema, organizados en tres niveles de causa - efecto.

Toda la información anterior fue analizada en una matriz de relaciones causales para identificar los "problemas resultado final", aquellos que no tienen capacidad de originar otros problemas (capacidad de arrastre) y a su vez poseen la mayor capacidad de ser originados por otros (capacidad de ser arrastrados).

Los resultados de la matriz de relaciones causales se procesaron en un cuadro similar al planteado por Schiller et al. (2001), mostrado en la Tabla 1, para determinar los indicadores que permitirán un monitoreo efectivo de los bienes y servicios esenciales del ecosistema, así como las metas socialmente definidas. Finalmente los indicadores fueron agrupados por temas siguiendo el Marco Ordenador PEIR de estado, presión, impacto y respuesta, y descritos detalladamente en fichas metodológicas para asegurar su monitoreo y replicabilidad (Anexo 1). 
Tabla 1. Para identificar indicadores.

\begin{tabular}{llll}
\hline \multicolumn{1}{c}{ Valor } & \multicolumn{1}{c}{$\begin{array}{c}\text { Punto de } \\
\text { valoración }\end{array}$} & \multicolumn{1}{c}{$\begin{array}{c}\text { Indicador } \\
\text { directo }\end{array}$} & $\begin{array}{l}\text { Indicador indirecto } \\
\text { o de diagnóstico }\end{array}$ \\
\hline $\begin{array}{l}\text { ¿Cuál es el bien } \\
\text { o servicio del } \\
\text { ecosistema, que } \\
\text { más interesa? }\end{array}$ & $\begin{array}{l}\text { ¿Qué se } \\
\text { necesita del } \\
\text { bien o } \\
\text { servicio } \\
\text { elegido? }\end{array}$ & $\begin{array}{l}\text { ¿Cómo se evalúa el } \\
\text { estado del bien o servicio } \\
\text { requerido? }\end{array}$ & $\begin{array}{l}\text { ¿Qué se debe medir } \\
\text { para tener una buena } \\
\text { evaluación del } \\
\text { servicio? }\end{array}$ \\
$\begin{array}{l}\text { ¿Qué meta se } \\
\text { busca alcanzar en } \\
\text { el ecosistema? }\end{array}$ & $\begin{array}{l}\text { necesita para } \\
\text { alcanzar la } \\
\text { meta }\end{array}$ & $\begin{array}{l}\text { ¿Cómo se evalúa el } \\
\text { estado del objetivo } \\
\text { planteado? }\end{array}$ & $\begin{array}{l}\text { ¿Qué se debe medir } \\
\text { para tener una buena } \\
\text { evaluación del } \\
\text { objetivo? }\end{array}$ \\
& & & \\
\hline
\end{tabular}

\section{Resultados y discusión}

Se elaboró el mapa de actores de la Reserva Nacional de Junín que muestra la relación existente entre los diferentes actores sociales de la zona (Figura 1). En el mapa se señala a las comunidades como el componente social con mayor influencia en la Reserva, y por lo tanto con mayor participación en este estudio, seguidas por la Jefatura de la Reserva Nacional de Junín del INRENA. La identificación de los actores sociales fue importante para el desarrollo del trabajo, pues se parte de sus necesidades críticas para

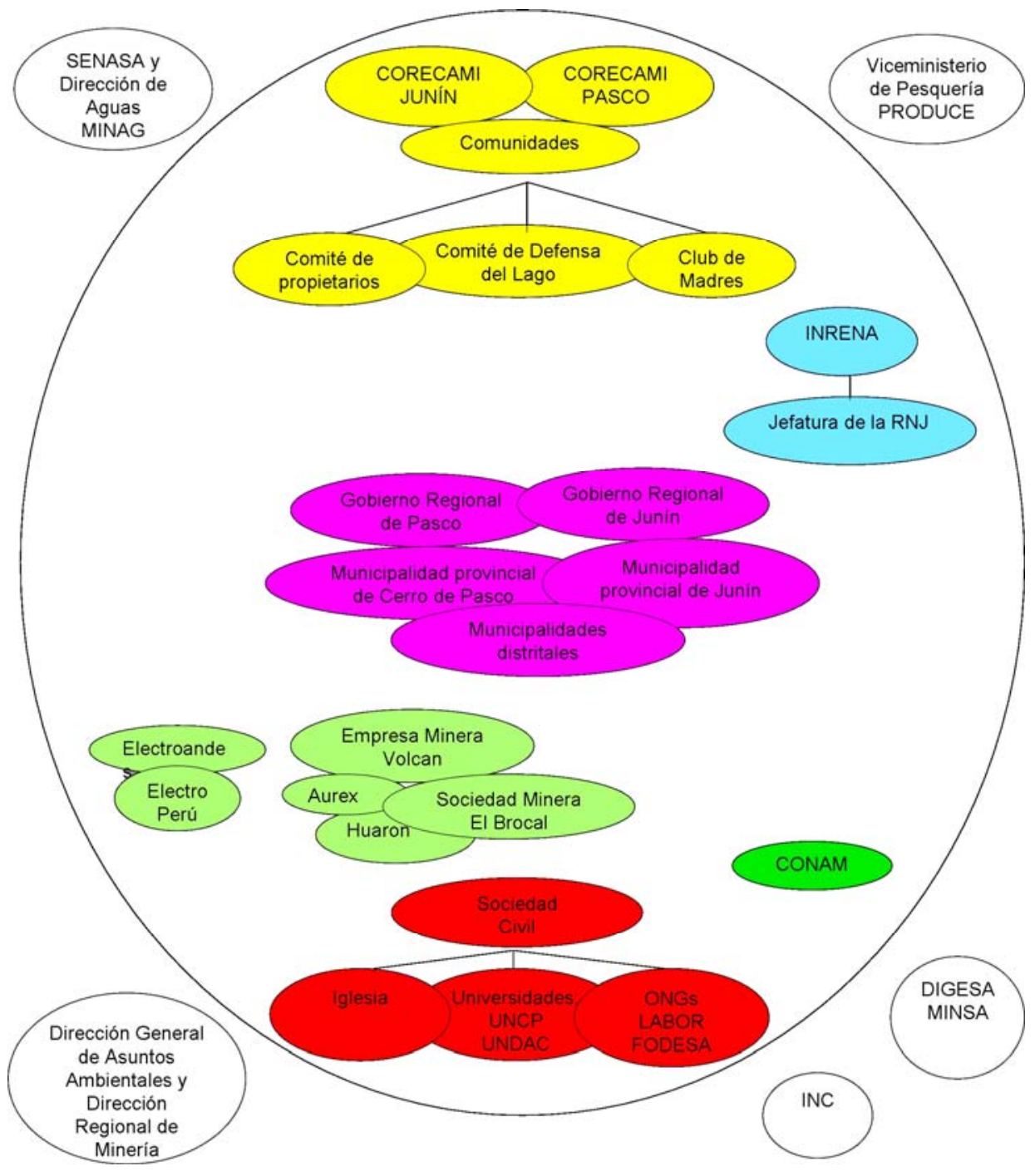

\section{Leyenda:}

Comunidades campesinas organizadas en el CORECAMI de Junín y Pasco, son los actores sociales con mayor presencia en la Reserva Nacional de Junín, por ser los usuarios directos de sus recursos.

INRENA: A través de su jefatura en Junín es la encargada de velar por el cumplimiento de los objetivos de creación de la RN Junín.

Autoridades Regionales $y$ Locales: Tienen responsabilidad de incluir en sus políticas aspectos que coadyuven a la conservación de la RN Junín.

Sector empresarial: Comprende a las empresas energéticas y mineras, las cuales son señaladas por los pobladores como la causa de varios de los problemas ambientales de la zona.

Sociedad Civil: Incluye al conjunto de instituciones que no usan directamente los recursos de la Reserva pero que están vinculados con su conservación, de alguna manera.

CONAM (Consejo Nacional del Ambiente): Es la Autoridad Ambiental Nacional, ente rector de la política ambiental del país, participa en la conservación de la Reserva a través del Comité de Gestión y Grupos Técnicos.

$\square$ Instituciones del Estado: Comprende el conjunto de instituciones que no trabajan directamente en la RN Junín, pero que a través de sus normas e instrumentos de gestión tienen influencia en la zona.

Figura 1. Mapa de Actores de la Reserva Nacional de Junín. 
llegar a los resultados colectivos y socialmente deseables (Walker et al., 2002) que permitirán plantear los indicadores de conservación de la Reserva.

El segundo aspecto tratado en esta investigación fue la identificación de los problemas más importantes por los que atraviesa la Reserva, los mismos que se muestran jerárquicamente ordenados en la Tabla 2.

Tabla 2. Problemas prioritarios de la Reserva Nacional de Junín

\begin{tabular}{lc}
\hline \multicolumn{1}{c}{ Problemas } & $\begin{array}{c}\text { Frecuencia } \\
\text { (\%) }\end{array}$ \\
\hline $\begin{array}{l}\text { 1. Degradación de calidad de los } \\
\text { pastos Contaminación del Lago Junín }\end{array}$ & 15 \\
$\begin{array}{l}\text { 2. Sobrepastoreo } \\
\text { 3. Extinción de especies como ranas } \\
\text { y el zambullidor de Junín } \\
\begin{array}{l}\text { 4. Deficiente servicio de desagüe y } \\
\text { acumulación de residuos sólidos } \\
\text { Otros (23 problemas distintos) }\end{array}\end{array}$ & 6 \\
\hline
\end{tabular}

Teniendo en cuenta cada problema prioritario se plantearon metas y objetivos socialmente deseables, que sirvieron de insumo para la identificación de los indicadores (Tabla 3)

La valoración participativa de los recursos que brinda la Reserva Nacional de Junín, según la cantidad de veces que fue nombrado cada uno de ellos, y las actividades a las que se dedican los habitantes de la zona se presentan en las Figuras 2 y 3,

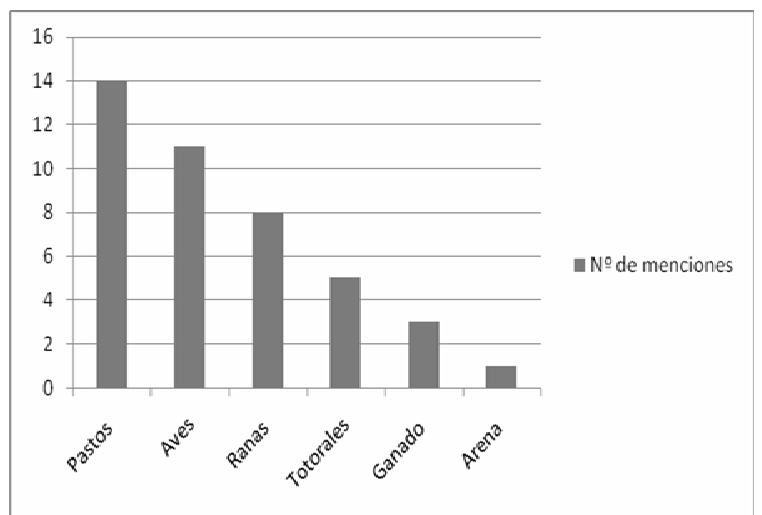

respectivamente.

Figura 2. Valoración de los recursos de la Reserva Nacional de Junín.

La Figura 2 muestra que los pastos son el recurso más importante para los habitantes de la zona, debido a que la gran mayoría de ellos se dedica a la actividad ganadera (Figura 3) y a que son la principal fuente
Tabla 3. Metas y objetivos socialmente deseables

\begin{tabular}{|c|c|}
\hline Metas & Objetivos \\
\hline $\begin{array}{l}\text { Mejorar la calidad de } \\
\text { los pastos de la } \\
\text { Reserva Nacional de } \\
\text { Junín }\end{array}$ & 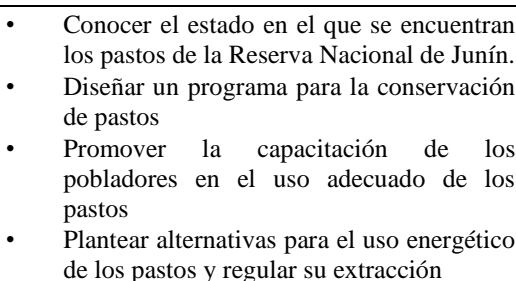 \\
\hline $\begin{array}{l}\text { Recuperar la calidad } \\
\text { del agua del Lago } \\
\text { Junín }\end{array}$ & 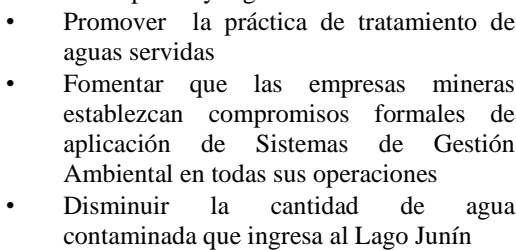 \\
\hline $\begin{array}{l}\text { Desarrollar técnicas } \\
\text { adecuadas de pastoreo }\end{array}$ & $\begin{array}{l}\text { - Diseñar un programa que permita un } \\
\text { manejo adecuado de pastoreo } \\
\text { - Mejorar técnicas de pastoreo }\end{array}$ \\
\hline $\begin{array}{l}\text { Recuperar especies en } \\
\text { vías de extinción } \\
\text { como el zambullidor y } \\
\text { rana de Junín }\end{array}$ & $\begin{array}{l}\text { - Elaborar un programa de protección y } \\
\text { manejo adecuado de los totorales } \\
\text { Evaluar la diversidad biológica del } \\
\text { ecosistema del Lago } \\
\text { - Otorgar protección a las aves residentes y } \\
\text { migratorias }\end{array}$ \\
\hline $\begin{array}{l}\text { Mejorar el servicio de } \\
\text { desagüe y reducir la } \\
\text { cantidad residuos } \\
\text { sólidos generados por } \\
\text { los habitantes de la } \\
\text { zona }\end{array}$ & $\begin{array}{l}\text { - Promover la instalación del servicio de } \\
\text { desagüe a todos los habitantes que viven cerca } \\
\text { de la Reserva Nacional de Junín } \\
\text { - Cumplir con la Ley General de Residuos } \\
\text { sólidos (Ley 27314) }\end{array}$ \\
\hline
\end{tabular}

energética de la Reserva, éstos vendrían a ser lo que Garibaldi \& Turner (2004) denominaron especies culturales clave y por lo tanto merecen especial atención. Se debe resaltar que los habitantes mencionan con mayor frecuencia los recursos sobre los que ha habido mayor difusión.

Además de hacer la valoración de los recursos naturales, se identificaron también los servicios esenciales que brinda el ecosistema, considerando que cada problema afecta directa o indirectamente a uno o más servicios que brinda la Reserva a las comunidades que habitan en ella. Los servicios identificados se presentan en la Tabla 4 (Millennium Ecosystem Assessment, 2003).

Para inferir acerca de la trayectoria que sigue la Reserva Nacional de Junín y definir indicadores que tomen en cuenta este aspecto fue importante identificar los conductores del ecosistema, definidos como aquellos factores que provocan los cambios en los ecosistemas y en los servicios que éstos prestan. Los conductores identificados se muestran en a Tabla 5 (Millennium Ecosystem Assessment, 2003).

A continuación se procedió a realizar un análisis de relaciones causales entre los problemas identificados en la Reserva y sus conductores. El resultado de este análisis fue la identificación de los problemas resultado final (Figura 4) en base a los 
cuales se priorizaron los indicadores de conservación de la Reserva.

Tabla 4. Servicios esenciales que brinda la Reserva Nacional de Junín.

\begin{tabular}{|c|c|}
\hline $\begin{array}{l}\text { Servicios de } \\
\text { suministro }\end{array}$ & $\begin{array}{ll}\text { - } & \text { Pastos } \\
\text { - } & \text { Calidad y cantidad de agua } \\
\text { - } & \text { Recursos hidrobiológicos } \\
\text { - } & \text { Especies de aves y anfibios } \\
\text { - } & \text { Totora }\end{array}$ \\
\hline $\begin{array}{l}\text { Servicios de } \\
\text { regulación }\end{array}$ & $\begin{array}{ll}\text { - } & \text { Control de enfermedades } \\
\text { - } & \text { Regulación del oxígeno disuelto en } \\
\text { - } & \text { Mantenimiento de la cadena trófica } \\
& \text { del Lago de Junín } \\
\text { - } & \text { Regulación del agua } \\
\text { - } & \text { Control de la erosión del suelo } \\
\text { - Ciclado de nutrientes en el suelo } \\
\text { - }\end{array}$ \\
\hline $\begin{array}{l}\text { Servicios } \\
\text { culturales }\end{array}$ & $\begin{array}{ll}\text { - } & \text { Paisaje adecuado para promover } \\
\text { el turismo } \\
\text { - } & \text { Ruinas arqueológicas } \\
\text { - } & \text { Escenario de hechos históricos } \\
\text { Posibilidades de enseñar a las } \\
\text { futuras generaciones las especies } \\
\text { típicas de la Reserva Nacional de } \\
\text { Junín }\end{array}$ \\
\hline $\begin{array}{l}\text { Servicios de } \\
\text { soporte }\end{array}$ & $\begin{array}{l}\text { - Suelos aptos para el crecimiento } \\
\text { de pastos y otros cultivos } \\
\text { - } \quad \text { Hábitat para especies de aves y } \\
\text { recursos hidrobiológicos }\end{array}$ \\
\hline
\end{tabular}

Con todos los insumos se identificó un juego de 24 indicadores (Tabla 6) para monitorear los procesos de conservación de la Reserva Nacional de Junín con énfasis en los problemas resultado final (degradación de la calidad de los pastos y la extinción de algunas especies de la fauna típica como el zambullidor y la rana de Junín, así como los conductores que motivaron su origen) teniendo en cuenta el logro de las metas socialmente determinadas y asegurando que el ecosistema continúe brindando sus servicios esenciales.

Los indicadores determinados se agruparon por temas y según el Marco Ordenador Presión, Estado, Impacto y Respuesta (Quiroga, 2001) donde los indicadores de estado son aquellos que muestran la situación actual en la que se encuentran las variables priorizadas en la Reserva; los indicadores de presión son los conductores del cambio de las variables priorizadas; los indicadores de impacto son las consecuencias de las presiones sobre las variables priorizadas o los servicios del ecosistema que se ven afectados por dichas presiones, y los indicadores de respuesta son aquellos que permiten evaluar el grado de avance o retroceso de las acciones del hombre tendientes a solucionar los problemas identificados o a alcanzar los objetivos planteados. Para asegurar su monitoreo y replicabilidad se describió la metadata de cada indicador en fichas metodológicas, una de las cuales se presenta como ejemplo en el Anexo 1.

\section{Conclusiones y recomendaciones}

Se ha determinado un juego de 24 indicadores para monitorear los procesos de conservación de la Reserva

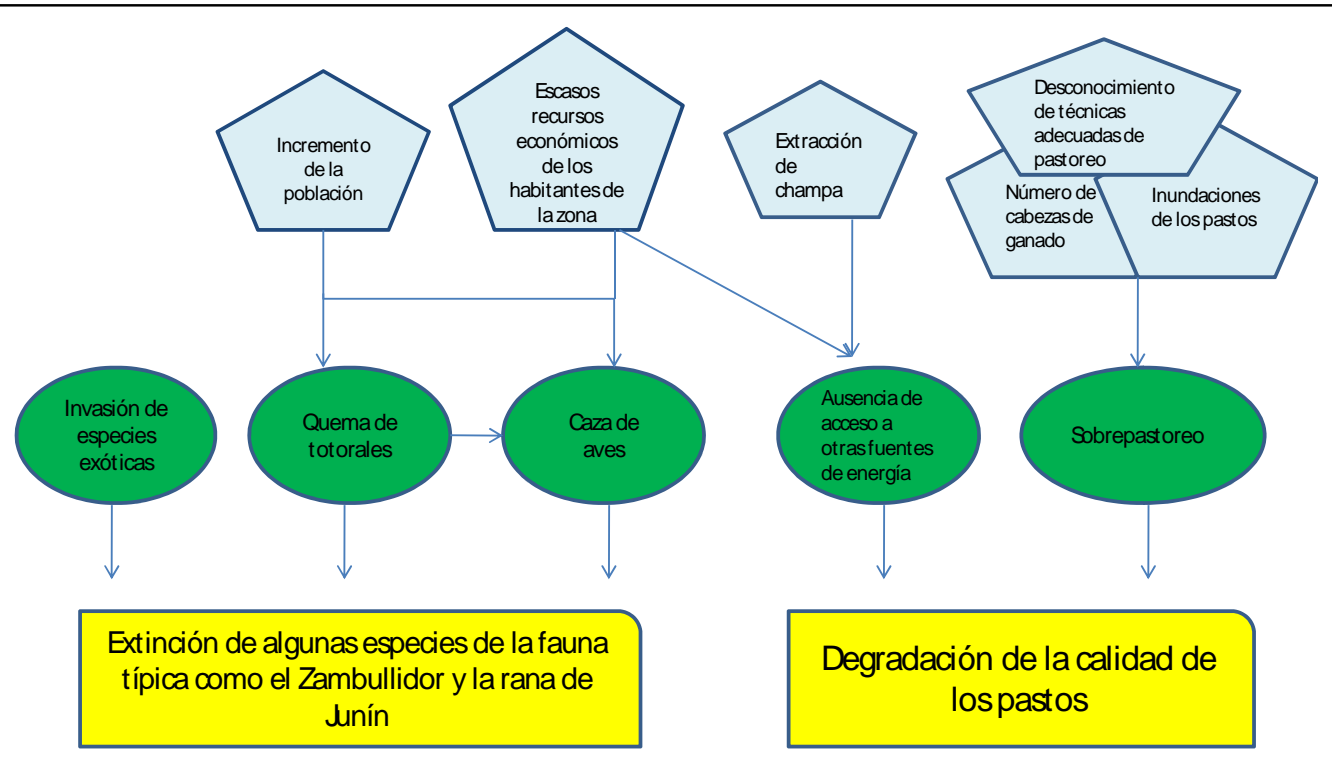

Figura 4. Problemas Resultado Final de la Reserva Nacional de Junín 
Nacional de Junín, teniendo en cuenta la participación de los actores sociales de la zona, los mismos que identificaron 5 problemas prioritarios que están relacionados a la degradación de calidad de los pastos, la contaminación del Lago Junín, el sobrepastoreo, la extinción de especies como ranas y el zambullidor de Junín, deficiente servicio de desagüe y acumulación de residuos sólidos. Siendo los problemas de degradación de calidad de los pastos y extinción de especies, los más importantes.

En base a cada problema se han identificado metas socialmente deseables y los servicios esenciales que brinda la Reserva, siendo la provisión de pastos el más importante y culturalmente clave para la zona. Estos aspectos, así como la identificación de conductores del ecosistema, se han tomado en cuenta en la determinación y priorización de los indicadores de conservación, los mismos que se sugiere que sean incluidos en los procesos de gestión de Área Natural Protegida del Perú, al estar estrechamente relacionados con la percepción de la sociedad que ocupa este espacio.

Se recomienda fortalecer el trabajo que se hace con las comunidades, debido a que éstas son consideradas los actores sociales más importantes de la Reserva y desarrollar acciones que mejoren la relación existente entre éstas y el INRENA. Asimismo, se debe fomentar un trabajo estrecho entre los actores sociales clave y los científicos para permitir una retroalimentación constante entre ambos grupos que permitan asegurar el éxito de cualquier medida que se implemente en beneficio de la Reserva.

Tabla 5. Conductores de la Reserva Nacional de Junín

\begin{tabular}{|c|c|}
\hline Conductores históricos: & $\begin{array}{ll}\text { - } & \text { Siembra de maca } \\
\text { - } & \text { Actividad Minera } \\
\text { - } & \text { Unstrucción de la represa de } \\
\text { - } & \text { Creación de la Reserva Nacional } \\
\text { de Junín } \\
\text { - Creación de cooperativas } \\
\text { comunales }\end{array}$ \\
\hline $\begin{array}{l}\text { Conductores políticos } \\
\text { relacionados a la gestión y } \\
\text { manejo de la Reserva } \\
\text { Nacional de Junín }\end{array}$ & $\begin{array}{l}\text { - } \quad \text { Ley de Áreas Naturales } \\
\text { Protegidas, Ley } 26834 \\
\text { - } \quad \text { Ley } 27642 \text { Emergencia Comisión } \\
\text { Multisectorial Descentralizad, } \\
\text { Plan } \\
\text { - Sistema de Manejo Ambiental } \\
\text { - Sostenible Chinchaycocha } \\
\text { Plan Maestro de la Reserva } \\
\text { Nacional de Junín }\end{array}$ \\
\hline Conductores internos & - $\quad$ Precipitación \\
\hline Conductores sociales & $\begin{array}{l}\text { - } \quad \text { Sobrextracción de ranas de Junín } \\
\text { - } \quad \text { Sobrepastoreo } \\
\text { - } \quad \text { Extracción de pastos (champa) } \\
\text { - } \quad \text { Inara uso energético } \\
\text { Sedimentos minerales acumulados en la } \\
\text { represa de Upamayo }\end{array}$ \\
\hline
\end{tabular}

\section{Agradecimientos}

Este trabajo fue posible gracias al apoyo de la Jefatura de la Reserva Nacional de Junín del INRENA y al señor Claudio Anibal Campos, ex presidente de la Comunidad de Villa Junín. Gracias también al señor David Solano por compartir el método de la matriz de relaciones causales que se usó esta investigación, al Ingeniero Ronald Medrano y al Biólogo Adrián Sánchez. Un agradecimiento muy especial al Antropólogo Arturo Caro y a la Asistenta Social Magda Vera, por facilitar el acercamiento a las ciencias sociales y el trabajo con las comunidades.

\section{Literatura citada}

Davic R.D. 2003. Linking Keystone Species and Functional Groups: A New Operational Definition of the Keystone Species Concept. Conservation Ecology. 7(1): 11. 1-14.

De Leo G.\& Levin S.1997. The Multifaceted Aspects Of Ecosystem Integrity. Conservation Ecology. 1: 1- 22.

Garibaldi A. \& Turner N. 2004. Cultural Keystone Species: Implications for Ecological Conservation and Restoration. Ecology and Society . 9(3): 1- 18.

Grupo de Páramos Punas y Jallqas del Perú: instituciones y acciones en Beneficio de Comunidades y Ecosistemas Altoandinos. 2002. Informe elaborado por el Centro de Información y Desarrollo Integral de Autogestión (CIDIAG), Centro Internacional de la Papa (CIP), Consejo Nacional del Ambiente (CONAM), Consejo Nacional de Camélidos Sudamericanos (CONACS), Consorcio para el Desarrollo Sostenible de la Ecorregión Andina (CONDEDSAN), Instituto de Gestión de Cuencas Hidrográficas, Instituto Nacional de recursos Naturales (INRENA), Instituto de Montaña (IM), Instituto del Bien Común (IBC), Fondo Nacional de Áreas Protegidas por el Estado (PROFONANPE) Universidad Nacional Agraria La Molina (UNALM). : 1-37.

Harwell M. 1999. A Framework for an Ecosystem Integrity Report Card. In Bioscience. : 1 -24.

INRENA. 2000. Plan Maestro de La Reserva Nacional de Junín. 2000. Instituto Nacional de Recursos Naturales del Perú. INRENA. : 1- 79.

Millar T. 1994. Ecología y Medio Ambiente. Grupo Editorial Iberoamérica. México. : 1- 867.

Millennium Ecosystem Assessment - (Program). 2003 Ecosystems and Human Well-being. A Framework for Assessment. World Resources Institute. USA. : 1 - 245.

Pintér L., Zahedi K., \& Cressman D.R. 2000. Capacitación para la Preparación de Evaluaciones e Informes Ambientales Integrados: Manual de Entrenamiento. Segunda edición. Instituto Internacional para el Desarrollo Sustentable (IIDS). Programa de las Naciones Unidas para el Medio Ambiente (PNUMA). Canadá. : 30 -64.

Quiroga R. 2001. Indicadores de Sostenibilidad Ambiental y de Desarrollo Sostenible: Estado del Arte y Perspectivas. CEPAL. División de Medio Ambiente y Asentamientos Humanos. Chile. : 1-116.

Schiller A., Huansaker C., Kane M., Wolfe A., Dale V., Glen Sutter G., Russel C., Pion G., Jensen M. \& Konar V. 2001 Communicating Ecological Indicators to Decision Makers and the Public. Conservation Ecology 5 (1): 1-24. 
Walker B., Carpenter S, Anderies J., Abel N., Cumming G.S., Janssen M., Lebel L., Norberg J., Peterson G.D. \& Pritchard R. 2002. Resilience Management in Social ecological Systems: a Working Hypothesis for a Participatory Approach. En Conservation Ecology. 6 (1): $1-20$.
Vásquez Monge E. \& Vegas Pozo J. 1985. Estudio SocioEconómico y Desarrollo Rural en las Comunidades Colindantes al Lago Junín. Universidad Nacional Mayor de San Marcos. Facultad de Ciencias Sociales. : 1- 47.

Tabla 6. Indicadores Priorizados para la Reserva Nacional de Junín.

\begin{tabular}{|c|c|c|c|c|}
\hline AREA TEMÁTICA & INDICADOR DE ESTADO & $\begin{array}{l}\text { INDICADOR DE } \\
\text { PRESIÓN }\end{array}$ & $\begin{array}{l}\text { INDICADOR DE } \\
\text { IMPACTO }\end{array}$ & $\begin{array}{l}\text { INDICADOR DE } \\
\text { RESPUESTA }\end{array}$ \\
\hline \multirow{4}{*}{ PASTOS } & $\begin{array}{l}\text { Superficie de pastos extraídos al } \\
\text { año con fines energéticos } \\
\text { (champeo) }\end{array}$ & $\begin{array}{l}\text { Número de permisos } \\
\text { para champeo } \\
\text { concedidos } \\
\text { anualmente }\end{array}$ & $\begin{array}{l}\text { \% de suelos perdidos al } \\
\text { año }\end{array}$ & $\begin{array}{l}\text { Superficie de áreas } \\
\text { forestadas con especies } \\
\text { nativas al año }\end{array}$ \\
\hline & $\begin{array}{l}\text { Superficie de pastizal disponible } \\
\text { para fines energéticos al año }\end{array}$ & & & \\
\hline & $\begin{array}{l}\text { Superficie de pastos disponibles } \\
\text { para el ganado, al año }\end{array}$ & $\begin{array}{l}\text { Número de cabezas } \\
\text { de ganado/ha de } \\
\text { pastizal, al año }\end{array}$ & $\begin{array}{l}\text { \% de suelos perdidos al } \\
\text { año }\end{array}$ & $\begin{array}{l}\text { Superficie de terreno con } \\
\text { manejo de pastos y sistema } \\
\text { de riego, al año }\end{array}$ \\
\hline & $\begin{array}{l}\text { Diversidad anual de plantas de la } \\
\text { Reserva Nacional de Junín }\end{array}$ & & & \\
\hline \multirow{2}{*}{ FAUNA } & $\begin{array}{l}\text { Número de individuos de } \\
\text { zambullidores de Junín al año }\end{array}$ & $\begin{array}{l}\text { Concentración anual } \\
\text { de metales en el Lago } \\
\text { Junín }\end{array}$ & & \\
\hline & $\begin{array}{l}\text { Número de individuos de ranas } \\
\text { de Junín al año }\end{array}$ & $\begin{array}{l}\text { Oxígeno disuelto en } \\
\text { el Lago de Junín, } \\
\text { anualmente }\end{array}$ & & \\
\hline \multirow{4}{*}{ POBLACION } & Densidad poblacional al año & $\begin{array}{l}\text { Tasa de crecimiento } \\
\text { poblacional al año }\end{array}$ & & \\
\hline & $\begin{array}{l}\text { Actividad económica per cápita } \\
\text { de los municipios asociados a la } \\
\text { Reserva Nacional de Junín al } \\
\text { año }\end{array}$ & & & \\
\hline & $\begin{array}{l}\text { Número de redes de desagüe } \\
\text { instaladas al año }\end{array}$ & & & \\
\hline & $\begin{array}{l}\text { Volumen de residuos sólidos } \\
\text { producidos al año }\end{array}$ & & & \\
\hline $\begin{array}{l}\text { CAPACITACIÓN Y } \\
\text { PARTICIPACIÓN } \\
\text { CIUDADANA }\end{array}$ & & & & $\begin{array}{l}\text { Número de personas que } \\
\text { han recibido capacitación } \\
\text { respecto al uso adecuado } \\
\text { de los pastos al año }\end{array}$ \\
\hline \multirow[t]{2}{*}{ LAGO DE JUNÍN } & $\begin{array}{l}\text { Nivel del Lago Junín dos veces } \\
\text { al año (época seca y época } \\
\text { lluviosa) }\end{array}$ & $\begin{array}{l}\text { Regulación del } \\
\text { volumen del agua en } \\
\text { la Represa de } \\
\text { Upamayo dos veces } \\
\text { por año (época seca y } \\
\text { época lluviosa) }\end{array}$ & $\begin{array}{l}\text { Superficie de pastos } \\
\text { perdidos por inundación } \\
\text { anualmente }\end{array}$ & \\
\hline & $\begin{array}{l}\text { Superficie de totorales en el Lago } \\
\text { de Junín al año }\end{array}$ & & & \\
\hline
\end{tabular}




\section{ANEXO 1}

Ficha Metodológica para Indicadores

\begin{tabular}{|c|c|c|c|}
\hline \multicolumn{4}{|l|}{ País: Perú } \\
\hline \multicolumn{4}{|c|}{ Nombre de la Región: Junín } \\
\hline \multicolumn{4}{|c|}{ Nombre del indicador: Número de individuos de zambullidores de Junín (Podiceps taczanowskii) } \\
\hline \multicolumn{4}{|c|}{ Descripción del indicador: Número de zambullidores de Junín en la Reserva de Junín } \\
\hline \multicolumn{4}{|c|}{ Unidad de medida: número } \\
\hline \multicolumn{4}{|l|}{ Fórmula: NZJzt =ni } \\
\hline \multicolumn{4}{|c|}{$\begin{array}{l}\text { Donde: NZJzt, es el número de Zambullidores de Junín i de la Reserva Nacional de Junín en un tiempo t } \\
\text { n, es el número total de Zambullidores de Junín i que se encuentran en el Lago de Junín }\end{array}$} \\
\hline \multicolumn{4}{|l|}{ Periodicidad : Anual } \\
\hline Cobertura geográfica & \multicolumn{3}{|c|}{ Área Natural Protegida } \\
\hline Disponibilidad & Desde: 1994 & \multicolumn{2}{|c|}{ Hasta: 2005} \\
\hline Facilidad de obtención & 1 Fácil & 2 Regular & 3 Difícil \\
\hline & & $\mathrm{X}$ & \\
\hline $\begin{array}{l}\text { Nombre de la fuente de } \\
\text { información }\end{array}$ & \multicolumn{3}{|c|}{ Tipo de fuente de información } \\
\hline $\begin{array}{l}\text { INRENA - RESERVA } \\
\text { NACIONAL DE JUNÍN }\end{array}$ & Censo & & \\
\hline \multicolumn{4}{|c|}{$\begin{array}{l}\text { Metodología de obtención del registro: Conteo total en espejo de agua en el mes de noviembre (nivel } \\
\text { mínimo) a través de transectos que crucen el Lago }\end{array}$} \\
\hline \multicolumn{4}{|l|}{ Responsable } \\
\hline \multicolumn{4}{|l|}{ Nombre: } \\
\hline \multicolumn{4}{|c|}{ Cargo: Jefe de la Reserva Nacional de Junín. } \\
\hline \multicolumn{4}{|l|}{ Dirección: } \\
\hline \multicolumn{4}{|l|}{ Correo electrónico: } \\
\hline Teléfono: & & & \\
\hline
\end{tabular}

\footnotetext{
${ }^{1}$ Universidad Nacional Agraria La Molina, Av. La Molina s/n, La Molina, Lima - Perú, Facultad de Ciencias, ccaro@lamolina.edu.pe

${ }^{2}$ Consejo Nacional del Ambiente, Av. Guardia Civil 205, San Borja , Lima - Perú, vmendoza@conam.gob.pe
} 